\title{
Tracking Control Design for Nonholonomic Mechanical Systems with Affine Constraints
}

\author{
Wei Sun ${ }^{1} \quad$ Yu-Qiang $\mathrm{Wu}^{2} \quad$ Zong-Yao $\mathrm{Sun}^{2}$ \\ ${ }^{1}$ School of Automation, Southeast University, Nanjing 210096, China \\ ${ }^{2}$ Institute of Automation, Qufu Normal University, Qufu 273165, China
}

\begin{abstract}
The trajectory tracking control is considered for nonholonomic mechanical systems with affine constraints and dynamic friction. A new state transformation is proposed to deal with affine constraints, and then an integral feedback compensation strategy is used to identify the dynamic friction. The proposed controller ensures that the output tracking errors converge to zero as $t \rightarrow \infty$. As an application, a detailed example is presented to illustrate the effectiveness of the control scheme.
\end{abstract}

Keywords: Tracking control, nonholonomic systems, affine constraints, constraint forces, integral feedback compensation.

\section{Introduction}

Nonholonomic constraints arise in many mechanical systems when there is a rolling or sliding contact, such as wheeled mobile robots, $n$-trailer systems, space robots, underwater vehicles, multi-fingered robotic hands and so on. Control of these systems has received considerable attention $^{[1-7]}$ due to the demand for control of the abovereferred systems.

The tracking problem for nonholonomic systems, as a much more interesting issue in practice, is to make the state of the closed-loop system track a given desired trajectory. For example, Wang et al. ${ }^{[8]}$ presented an adaptive robust control strategy for a class of mechanical systems with both holonomic and nonholonomic constraints. Based on physical properties, adaptive robust motion/force control for wheeled inverted pendulums is investigated in [9]. For mobile manipulators under both holonomic and nonholonomic constraints, the authors of [10] and [11] proposed state-feedback control strategies by introducing an appropriate state transformation, and adaptive robust outputfeedback force/motion control strategies, respectively. It is worth pointing out that tracking control of nonholonomic systems with linear constraints $(J(q) \dot{q}=0)$ has been widely studied $^{[8-14]}$ to date. In fact, there is another large class of constraints which are affine in velocities, called affine constraints $^{[15-17]}(J(q) \dot{q}=A(q))$, such as a boat on a running river with the varying stream, ball on rotating table with invariable angular velocity, under-actuated mechanical arm, etc. Kai ${ }^{[15]}$ defined rheonomous affine constraints and explained a geometric representation method for them, then derived a necessary and sufficient condition for completing

Brief Paper

Brief Paper

This work was supported by National Natural Science Foundation of China (Nos. 61273091, 61004013 and 61304059), Ph. D. Programs Foundation of Ministry of Education of China, and Fundamental Research Funds for the Central Universities (No. CXLX12_0096). nonholonomicity of the rheonomous affine constraints. In [16], Kai et al. derived very good results about nonholonomic dynamic systems with affine constraints. To be specific, he got several preliminary properties of this system, investigated local accessibility and local controllability based on both Sussmann's theorem and linear approximation approaches, and derived conditions for local asymptotic stabilizability by linear state feedback and nonlinear smooth state feedback at last. However, tracking control for such systems has not been investigated until now. Hence, researching tracking control for such mechanical systems is an innovatory and significative work.

A new continuous control mechanism compensated for uncertainty in a class of high-order, multiple-input multipleoutput nonlinear systems, was presented in [18]. Based on this control strategy, Makkar et al. considered modeling and compensation for parameterizable friction effects for a class of mechanical systems in [19]. In [20], Patre et al. developed a novel adaptive nonlinear control design which achieves modularity between the controller and the adaptive update law. Based on this compensatory strategy for the uncertainties and the asymptotic tracking idea for uncertain multi-input nonlinear systems, the trajectory tracking control for nonholonomic mechanical systems with affine constraints and dynamic friction is considered in this paper.

The rest of this paper is organized as follows. System description and control design are given in Section 2. Section 3 addresses the main results. For the application, a practical example is considered in Section 4. Section 5 gives some concluding remarks.

Notations. $\|x\|$ denotes the Euclidean norm of $x$; $\operatorname{sgn}(\cdot)$ denotes the standard signum function; we say that $x(t) \in \mathcal{L}_{\infty}$ when $\|x\|_{\infty} \triangleq \sup _{t \geqslant 0}|x(t)|$ exists; a continuous function $h: \mathbf{R}^{+} \rightarrow \mathbf{R}^{+}$is said to be a $\mathcal{K}$ function if it is strictly increasing and vanishes at zero. For simplicity, sometimes the arguments of functions are dropped. 


\section{System description and control de- sign}

\subsection{Dynamics model}

In this paper, we consider a class of nonholonomic mechanical systems described by Euler-Lagrangian equation:

$$
M(q) \ddot{q}+V(q, \dot{q}) \dot{q}+G(q)+F(q, \dot{q})=\tau+f
$$

where $q=\left[q_{1}, \cdots, q_{n}\right]^{\mathrm{T}} \in \mathbf{R}^{n}$ is the generalized coordinates, and $\dot{q}, \ddot{q} \in \mathbf{R}^{n}$ represent the generalized velocity vector and acceleration vector, respectively; $M(q) \in \mathbf{R}^{n \times n}$ is the inertia matrix; $V(q, \dot{q}) \dot{q} \in \mathbf{R}^{n}$ presents the centripetal, Coriolis forces; $G(q) \in \mathbf{R}^{n}$ represents the gravitational force; $F(q, \dot{q}) \in \mathbf{R}^{n}$ represents the friction force; $\tau \in \mathbf{R}^{n}$ is the vector of control input; $f=J(q) \lambda \in \mathbf{R}^{n}$ denotes the vector of constraint force, where $J(q) \in \mathbf{R}^{n \times m}$ is constraint matrix, and $\lambda \in \mathbf{R}^{m}$ is Lagrangian multiplier corresponding to $m$ nonholonomic affine constraints which are represented by analytical relations between the generalized coordinates $q$ and velocity vectors $\dot{q}$, and can be written as:

$$
J^{\mathrm{T}}(q) \dot{q}=A(q)
$$

where $J(q)=\left[j_{1}(q), \cdots, j_{m}(q)\right] \in \mathbf{R}^{n \times m}$ is of full rank, and $A(q)=\left[a_{1}(q), \cdots, a_{m}(q)\right]^{\mathrm{T}} \in \mathbf{R}^{m}$ is a known vector function.

Remark 1. It is worth emphasizing that the system studied in this paper is more general than that in some existing literatures such as $[9,12,14]$, where dynamic equation satisfies the classical linear constraint. In fact, by taking $A(q)=0,(2)$ transforms to linear constraints, whose tracking problem has received considerable attention.

The subsequent development is based on the assumption that $M(q), V(q, \dot{q}), G(q)$ are known and bounded if their elements are all bounded. Moreover, in order to facilitate the subsequent design and analysis, the following assumptions will be exploited:

Assumption 1. If $q$ and $\dot{q}$ are bounded, the inertia matrix $M(q)$ satisfies $\frac{\partial M(q)}{\partial q} \in \mathcal{L}_{\infty}$.

Assumption 2. The dynamic friction term $F(q, \dot{q})$ satisfies $F(q, \dot{q}) \in \mathcal{L}_{\infty}, \dot{F}(q, \dot{q}) \in \mathcal{L}_{\infty}, \ddot{F}(q, \dot{q}) \in \mathcal{L}_{\infty}$, if their elements are bounded.

\subsection{State transformation}

This part mainly focuses on reducing the number of state variables which provide motion complying with the affine constraints.

It is easy to find a full-rank matrix $S(q) \in \mathbf{R}^{n \times(n-m)}$ satisfying

$$
J^{\mathrm{T}}(q) S(q)=0
$$

Define $\xi(t)=[q,-t]^{\mathrm{T}}$, then $(2)$ can be expressed concisely as

$$
\left[\begin{array}{ll}
J^{\mathrm{T}}(q) & A(q)
\end{array}\right] \dot{\xi}(t)=0 .
$$

Let

$$
E(q)=\left[\begin{array}{cc}
S(q) & \eta(q) \\
0 & -1
\end{array}\right] \in \mathbf{R}^{(n+1) \times(n-m+1)}
$$

where $\eta(q) \in \mathbf{R}^{n}$ satisfies $J^{\mathrm{T}}(q) \eta(q)=A(q)$. One can deduce that $E$ is a full-rank matrix and satisfies

$$
\left[\begin{array}{ll}
J^{\mathrm{T}}(q) & A(q)
\end{array}\right] E(q)=0 .
$$

From (4) and (5), we know that there exists a $(n-m+1)$ dimensional vector $\dot{\bar{z}}=\left[\dot{\bar{z}}_{n-m}^{\mathrm{T}}, \dot{\bar{z}}_{n-m+1}\right]^{\mathrm{T}}$ such that $\dot{\xi}=E \dot{\bar{z}}$, that is

$$
\left[\begin{array}{c}
\dot{q} \\
-1
\end{array}\right]=\left[\begin{array}{cc}
S(q) & \eta(q) \\
0 & -1
\end{array}\right]\left[\begin{array}{c}
\dot{\bar{z}}_{n-m}^{\mathrm{T}} \\
\dot{\bar{z}}_{n-m+1}
\end{array}\right]
$$

which implies $\dot{\bar{z}}_{n-m+1}=1$. For convenience, define $z \triangleq$ $\bar{z}_{n-m} \in \mathbf{R}^{n-m}$. In view of the relationship (6), the generalized velocity vectors can be written as

$$
\dot{q}=S(q) \dot{z}+\eta(q) .
$$

It is clear that $z$ corresponds to the internal state variable.

Substituting (7) into (1), pre-multiplying $S^{\mathrm{T}}(q)$ on both sides of it, and using $J^{\mathrm{T}}(q) S(q)=0$, the dynamics of the mechanical system made up by (1) and (2) can be described clearly as

$$
M_{1}(q) \ddot{z}+V_{1}(q, \dot{q}) \dot{z}+G_{1}(q, \dot{q})+F_{1}(q, \dot{q})=\tau_{1}
$$

where $M_{1}(q)=S^{\mathrm{T}}(q) M(q) S(q), \quad V_{1}(q, \dot{q})=S^{\mathrm{T}}(q)$ $(M(q) \dot{S}(q)+V(q, \dot{q}) S(q)), \quad G_{1}(q, \dot{q})=S^{\mathrm{T}}(q)(M(q) \dot{\eta}(q)$ $+V(q, \dot{q}) \eta(q)+G(q)), \tau_{1}=S^{\mathrm{T}}(q) \tau, F_{1}(q, \dot{q})=S^{\mathrm{T}}(q)$ $F(q, \dot{q})$. fies

Property $\mathbf{1}^{[21]}$. The matrix $M_{1}$ is symmetric and satis-

$$
a\|x\|^{2} \leqslant x^{\mathrm{T}} M_{1}(x) x \leqslant \bar{a}(\|x\|)\|x\|^{2}
$$

where $a$ is a known positive constant, $\bar{a}(x)$ is a known positive function.

Remark 2. The above transformations consist of (3) and (7) ensure that the transformed system (8) still satisfies constraint equation (2), and possesses the practical physical meaning, for further detail, see $[8,9]$. This can also be confirmed by the practical example in Section 4 .

Remark 3. The aforementioned transform method differs from the traditional ones in [8-11]. More specifically, when the affine constraints are imposed on the mechanical system, it is difficult to find linearly independent vector fields to proceed with a simple diffeomorphism transformation for canceling the constraint forces in dynamic equations. Hence, we present the aforementioned transform to achieve this goal.

\subsection{Control design}

The control objective of this paper can be specified as follows. Given the desired trajectories $z_{d}(t)$ and $\dot{z}_{d}(t)$ which are assumed to be bounded and should satisfy constraint (2). In addition, $\ddot{z}_{d}(t)$ and $\dddot{z}_{d}(t)$ are assumed to exist and be bounded. We determine a control law such that the internal state $z(t)$ and $\dot{z}(t)$ are globally bounded and the output tracking error

$$
e_{1}(t)=z_{d}(t)-z(t) .
$$

And its time derivative $\dot{e}_{1}(t)$ converges to zero. 
To achieve the desired control objective, the following filtered tracking errors ${ }^{[19,20]}$ are defined as

$$
\left\{\begin{aligned}
e_{2}(t) & =\dot{e}_{1}(t)+\alpha_{1} e_{1}(t) \\
\rho(t) & =\dot{e}_{2}(t)+\alpha_{2} e_{2}(t)
\end{aligned}\right.
$$

where $e_{2}(t), \rho(t) \in \mathbf{R}^{n-m}$, and $\alpha_{1}>0, \alpha_{2}>0$ are design constants. The filtered tracking error $\rho(t)$ is not measurable because its expression depends on $\ddot{z}(t)$. In view of (8) and (9), pre-multiplying (10) by $M_{1}$, the following expression can be obtained

$$
\begin{aligned}
M_{1}(q) \rho(t)= & M_{1}(q) \ddot{z}_{d}(t)+V_{1}(q, \dot{q}) \dot{z}(t)+ \\
& G_{1}(q, \dot{q})+F_{1}(q, \dot{q})-\tau_{1}+ \\
& \alpha_{1} M_{1}(q) \dot{e}_{1}(t)+\alpha_{2} M_{1} e_{2}(t) .
\end{aligned}
$$

Based on (11), the control torque input is designed as

$$
\begin{aligned}
\tau_{1}= & M_{1}(q) \ddot{z}_{d}(t)+V_{1}(q, \dot{q}) \dot{z}(t)+G_{1}(q, \dot{q})+ \\
& \alpha_{1} M_{1}(q) \dot{e}_{1}(t)+\alpha_{2} M_{1} e_{2}(t)+u(t)
\end{aligned}
$$

where $u(t) \in \mathbf{R}^{n-m}$ denotes a subsequently designed control term. Substituting (12) into (11), one can get

$$
M_{1}(q) \rho(t)=F_{1}(q, \dot{q})-u(t) .
$$

It is evident that if $\rho(t) \rightarrow 0$, then $u(t)$ will identify the friction model $F_{1}(q, \dot{q})$. Hence, the next objective is to design the control term $u(t)$ to ensure $\rho \rightarrow 0$. To facilitate the design of $u(t)$, differentiating (13) yields:

$$
M_{1}(q) \dot{\rho}(t)=\dot{F}_{1}(q, \dot{q})-\dot{u}(t)-\dot{M}_{1}(q) \rho(t) .
$$

Similar to [19], $u(t)$ is designed as

$$
\begin{aligned}
u(t)= & \left(k_{s}+1\right) e_{2}(t)-\left(k_{s}+1\right) e_{2}(0)+ \\
& \int_{0}^{t}\left(\left(k_{s}+1\right) \alpha_{2} e_{2}(\tau)+\beta \operatorname{sgn}\left(e_{2}(\tau)\right)\right) \mathrm{d} \tau
\end{aligned}
$$

where $k_{s} \in \mathbf{R}$ is control gain, and $\beta \in \mathbf{R}$ is a positive constant which will be specified later. $u(t)$ expressed in (15) does not depend on the unmeasurable filtered tracking error term $\rho$, but its time derivative of $u(t)$ can be expressed as a function of $\rho(t)$. Taking the time derivative of (15), one can get

$$
\dot{u}(t)=\left(k_{s}+1\right) \rho(t)+\beta \operatorname{sgn}\left(e_{2}(t)\right) .
$$

Substituting (16) into (14), the following closed-loop error system can be obtained:

$$
\begin{aligned}
M_{1}(q) \dot{\rho}(t)= & -\frac{1}{2} \dot{M}_{1}(q) \rho(t)-\left(k_{s}+1\right) \rho(t)-e_{2}(t)- \\
& \beta \operatorname{sgn}\left(e_{2}(t)\right)+\Theta(q, \dot{q}, t)
\end{aligned}
$$

where $\Theta(q, \dot{q}, t)=\dot{F}_{1}(q, \dot{q})-\frac{1}{2} \dot{M}_{1}(q) \rho(t)+e_{2}(t)$. We define

$$
\Theta_{d}=\frac{\partial F_{1}\left(z_{d}, \dot{z}_{d}\right)}{\partial z_{d}} \dot{z}_{d}(t)+\frac{\partial F_{1}\left(z_{d}, \dot{z}_{d}\right)}{\partial \dot{z}_{d}} \ddot{z}_{d}(t) .
$$

In view of Assumption 2 and Remark 1, it is noted that $\dot{z}_{d}, \ddot{z}_{d}$ and $\dddot{z}_{d}$ are all bounded. Hence, one can find known positive constants $B_{1}$ and $B_{2}$, such that

$$
\left\|\Theta_{d}\right\| \leqslant B_{1}, \quad\left\|\dot{\Theta}_{d}\right\| \leqslant B_{2} .
$$

Define $\tilde{\Theta}(t)=\Theta(t)-\Theta_{d}(t)$, then the closed-loop error system (17) can be rewritten as

$$
\begin{aligned}
M_{1}(q) \dot{\rho}(t)= & -\frac{1}{2} \dot{M}_{1}(q) \rho(t)-\left(k_{s}+1\right) \rho(t)-e_{2}(t)- \\
& \beta \operatorname{sgn}\left(e_{2}(t)\right)+\tilde{\Theta}(t)+\Theta_{d}(t) .
\end{aligned}
$$

\section{Main results}

Now, we are ready to present the following theorem, which summarizes the main results of the paper.

Theorem 1. Consider the nonholonomic mechanical system described by (1) and (2), subject to Assumptions 1 and 2 , if we select parameter $\beta$ satisfying $\beta>B_{1}+\frac{1}{\alpha_{2}} B_{2}$, then the controller given in (12) and (15) ensure that all the signals of closed-loop system are bounded and the tracking errors $e_{1}(t), \dot{e}_{1}(t) \rightarrow 0$ as $t \rightarrow \infty$.

Proof. Let $D \in \mathbf{R}^{3(n-m)+1}$ be a domain containing $y(t)=0$, where $y(t) \in \mathbf{R}^{3(n-m)+1}$ is defined as $y(t)=\left[x^{\mathrm{T}}(t), \sqrt{P(t)}^{\mathrm{T}}, x(t) \in \mathbf{R}^{3(n-m)}\right.$ is defined as $x(t)=\left[e_{1}^{\mathrm{T}}(t), e_{2}^{\mathrm{T}}(t), \rho^{\mathrm{T}}(t)\right]^{\mathrm{T}}$, and the function $P(t) \in \mathbf{R}$ is defined as

$$
P(t)=\beta\left\|e_{2}(0)\right\|-e_{2}(0)^{\mathrm{T}} \Theta_{d}(0)-\int_{0}^{t} L(\tau) \mathrm{d} \tau
$$

where the auxiliary function $L(t)$ is defined as

$$
L(t)=\rho^{\mathrm{T}}\left(\Theta_{d}(t)-\beta \operatorname{sgn}\left(e_{2}(t)\right)\right) .
$$

Then, if $\beta>B_{1}+\frac{1}{\alpha_{2}} B_{2}$, with manipulations similar to Appendix A in [18], the following inequality can be obtained

$$
\int_{0}^{t} L(\tau) \mathrm{d} \tau \leqslant \beta\left\|e_{2}(0)\right\|-e_{2}(0)^{\mathrm{T}} \Theta_{d}(0) .
$$

Hence, one can deduce $P(t) \geqslant 0$.

Choose a candidate Lyapunov function ${ }^{[19,20]}$ as

$$
\begin{aligned}
V(t)= & e_{1}^{\mathrm{T}}(t) e_{1}(t)+\frac{1}{2} e_{2}^{\mathrm{T}}(t) e_{2}(t)+ \\
& \frac{1}{2} \rho^{\mathrm{T}}(t) M_{1}(q) \rho(t)+P(t) .
\end{aligned}
$$

Taking the time derivative of $V(t)$ along the solutions of (8), and substituting (9), (10) and (18) into it, we have

$$
\begin{aligned}
\dot{V}(t)= & 2 e_{1}^{\mathrm{T}}(t) \dot{e}_{1}(t)+e_{2}^{\mathrm{T}}(t) \dot{e}_{2}(t)+\rho^{\mathrm{T}}(t) M_{1}(q) \dot{\rho}(t)+ \\
& \frac{1}{2} \rho^{\mathrm{T}}(t) \dot{M}_{1}(q) \rho(t)+\dot{P}(t) \leqslant \\
& -2 \alpha_{1}\left\|e_{1}(t)\right\|^{2}-\alpha_{2}\left\|e_{2}(t)\right\|^{2}-\left(k_{s}+1\right)\|\rho(t)\|^{2}+ \\
& 2 e_{1}^{\mathrm{T}}(t) e_{2}(t)+\rho^{\mathrm{T}}(t) \tilde{\Theta} .
\end{aligned}
$$

Since $\Theta$ is continuously differentiable, according to the mean value theorem, we can get the upper bound of $\tilde{\Theta}$ as follows $^{[18]}$ :

$$
\|\tilde{\Theta}\| \leqslant \varphi(\|x(t)\|)\|x(t)\|
$$

where $\varphi(\|x(t)\|)$ is an appropriate $\mathcal{K}$ function. In view of $2 e_{1}^{\mathrm{T}}(t) e_{2}(t) \leqslant\left\|e_{1}(t)\right\|^{2}+\left\|e_{2}(t)\right\|^{2}, \dot{V}(t)$ can be simplified as $\dot{V}(t) \leqslant-\lambda\|x(t)\|^{2}-\left(k_{s}\|\rho(t)\|^{2}-\varphi(\|x(t)\|)\|\rho(t)\|\|x(t)\|\right)$ 
where $\lambda=\min \left\{2 \alpha_{1}-1, \alpha_{2}-1,1\right\}$, and $\alpha_{1}, \alpha_{2}$ satisfy $\alpha_{1}>$ $\frac{1}{2}, \alpha_{2}>1$.

Completing the squares for third term in above inequality, one can easily get

$$
\varphi(\|x(t)\|)\|\rho(t)\|\|x(t)\| \leqslant k_{s}\|\rho(t)\|^{2}+\frac{\varphi^{2}(\|x(t)\|)\|x(t)\|^{2}}{4 k_{s}} .
$$

With this inequality in mind, inequality (20) reduces to

$$
\dot{V}(t) \leqslant-\lambda\|x(t)\|^{2}+\frac{\varphi^{2}(\|x(t)\|)\|x(t)\|^{2}}{4 k_{s}} .
$$

Now we define a compact set:

$$
N_{1}=\left\{y(t) \in \mathbf{R}^{3(n-m)+1} \mid\|y(t)\| \leqslant \varphi^{-1}\left(2 \sqrt{\lambda k_{s}}\right)\right\} .
$$

The inequality $(21)$ can be used to show that $V(t) \leqslant$ $V(0)$ in $N_{1}$, hence, all the the signals $e_{1}(t), e_{2}(t), \rho(t)$ on the right-hand side of function (19) are bounded in $N_{1}$. From the definition of $e_{1}(t), e_{2}(t)$ and $\rho(t)$, we can further get $\dot{e}_{1}(t), \dot{e}_{2}(t) \in \mathcal{L}_{\infty}$ in $N_{1}$. The assumption that $z_{d}(t), \dot{z}_{d}(t), \ddot{z}_{d}(t)$ are bounded can be used to conclude that $z(t), \dot{z}(t), \ddot{z}(t) \in \mathcal{L}_{\infty}$ are in $N_{1}$. We know $M_{1}(q), V_{1}(q, \dot{q})$, $G_{1}(q)$ are all bounded in $N_{1}$. Hence, $\tau_{1} \in \mathcal{L}_{\infty}$ is in $N_{1}$.

Now, let $N_{2} \subset N_{1}$ denote a set defined as follows:

$$
N_{2}=\left\{y(t) \subset N_{1} \mid \delta_{2}(y(t))\|y(t)\|^{2}<\delta_{1}\left(\varphi^{-1}\left(2 \sqrt{\lambda k_{s}}\right)\right)^{2}\right\}
$$

where $\delta_{1}=\frac{1}{2} \min \{1, a\}, \delta_{2}(y(t))=\max \left\{1, \frac{1}{2} \bar{a}(y(t))\right\}$, and the definition of $a$ and $\bar{a}(y(t))$ have been given in Property 1 . From (21), one can obtain that there must exist a positive function $U(y(t))=c\|x(t)\|^{2}$, such that

$$
\dot{V}(t) \leqslant-U(y(t))
$$

Invariance-like Theorem ${ }^{[22]}$ can be used to show that

$$
U(y(t))=c\|x(t)\|^{2} \rightarrow 0, \quad t \rightarrow \infty, \quad \forall y(0) \in N_{2}
$$

Based on the definitions of $x(t)$, one has $e_{1}(t), e_{2}(t) \rightarrow 0$ as $t \rightarrow \infty, \forall y(0) \in N_{2}$. From (10), we finally get $\dot{e}_{1}(t) \rightarrow 0$ as $t \rightarrow \infty, \forall y(0) \in N_{2}$.

\section{Simulation}

Consider a boat with payload on a running river ${ }^{[17]}$ (see Fig. 1). The $x$-axis and $y$-axis denote the transverse direction and the downstream direction of the river, respectively. Here, we suppose the stream of the river only depends on transverse position $x$ in the simulation. According to the motion of boat on the river, one can get the following kinematic equations:

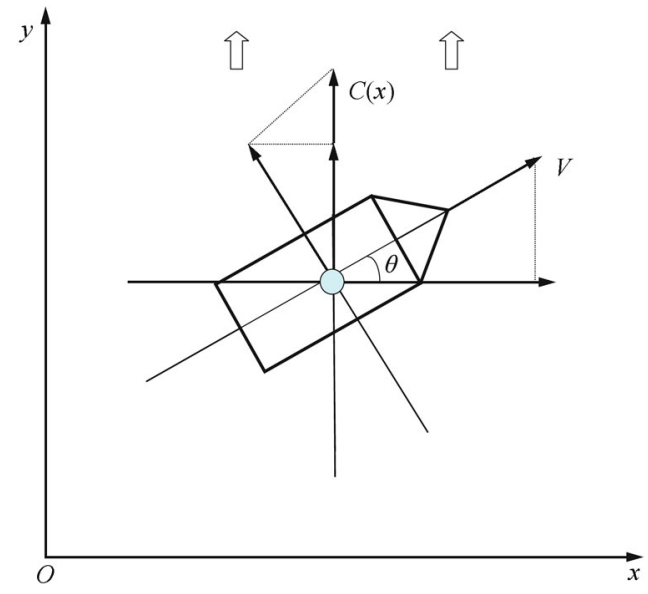

Fig. 1 Boat on a running river

$$
\left\{\begin{array}{c}
\dot{x}=V \cos \theta-C(x) \cos \theta \sin \theta \\
\dot{y}=V \sin \theta+C(x) \cos ^{2} \theta
\end{array}\right.
$$

where $C(x)$ denotes the stream of the river. After some simple calculations, the affine constraints can be obtained as

$$
\dot{y} \cos \theta-\dot{x} \sin \theta=C(x) \cos \theta
$$

where $C(x) \cos \theta$ corresponds to $A(q)$ in (2).

After imposing the constraint forces, and noting $V(q, \dot{q}) \dot{q}=0, G(q)=0$, one can get the following dynamic equation:

$$
M(q) \ddot{q}+F(q, \dot{q})=\tau+J(q) \lambda
$$

where $q=[y, x, \theta]^{\mathrm{T}}, M(q)=\operatorname{diag}\{m, m, I\}$ and $J^{\mathrm{T}}(q)=$ $\left[\cos q_{3},-\sin q_{3}, 0\right], m$ is the mass of the boat, and $I$ is the inertia of the boat. For the sake of simplicity, select $m=$ $1 \mathrm{~kg}, I=1 \mathrm{~kg} \cdot \mathrm{m}^{2}, C\left(q_{2}\right)=q_{2}, F(q)=\left[\cos q_{3}, 0,0\right]^{\mathrm{T}}$.

We select

$$
\begin{aligned}
& S(q)=\left[\begin{array}{cc}
\tan q_{3} & 0 \\
1 & 0 \\
0 & 1
\end{array}\right] \\
& \eta(q)=\left[\begin{array}{lll}
q_{2} & 0 & 0
\end{array}\right]^{\mathrm{T}} .
\end{aligned}
$$

It follows from the transformation (7) that

$$
\left\{\begin{array}{l}
\dot{q}_{1}=\dot{z}_{1} \tan q_{3}+q_{2} \\
\dot{q}_{2}=\dot{z}_{1} \\
\dot{q}_{3}=\dot{z}_{2}
\end{array}\right.
$$

Substituting (23) into (22), pre-multiplying both sides of it by $S^{\mathrm{T}}(q)$, and using $J^{\mathrm{T}}(q) S(q)=0$, one can get

$$
\begin{gathered}
{\left[\begin{array}{cc}
1+\tan ^{2} \theta & 0 \\
0 & 1
\end{array}\right]\left[\begin{array}{c}
\ddot{z}_{1} \\
\ddot{z}_{2}
\end{array}\right]+\left[\begin{array}{cc}
\frac{\sin \theta}{\cos ^{3} \theta} & 0 \\
0 & 0
\end{array}\right]\left[\begin{array}{c}
\dot{z}_{1} \\
\dot{z}_{2}
\end{array}\right]+} \\
{\left[\begin{array}{c}
\dot{q}_{2} \tan \theta \\
0
\end{array}\right]+\left[\begin{array}{c}
\sin \theta \\
0
\end{array}\right]=\tau_{1}}
\end{gathered}
$$


For the given $J(q), S(q)$ and $\eta(q)$, the desired trajectory $q_{d}=\left[\sin t-\cos t, \sin t, \frac{\pi}{4}\right]^{\mathrm{T}}$ satisfies kinematic constraint $J^{\mathrm{T}}\left(q_{d}\right) \dot{q}_{d}=A\left(q_{d}\right)$ and transform $\dot{q}_{d}=S\left(q_{d}\right) \dot{z}_{d}+\eta\left(q_{d}\right)$ with $z_{d}=\left[\sin t, \frac{\pi}{4}\right]^{\mathrm{T}}$. The control objective is to determine a feedback controller so that $z$ follows $z_{d}$ and $\dot{z}$ follows $\dot{z}_{d}$, respectively.

Based on the previous control design (12) and (15), we get the actual controller as

$$
\begin{aligned}
\tau_{1}= & {\left[\begin{array}{cc}
1+\tan ^{2} \theta & 0 \\
0 & 1
\end{array}\right]\left[\begin{array}{l}
\ddot{z}_{1 d} \\
\ddot{z}_{2 d}
\end{array}\right]+} \\
& {\left[\begin{array}{cc}
\frac{\sin \theta}{\cos ^{3} \theta} & 0 \\
0 & 0
\end{array}\right]\left[\begin{array}{l}
\dot{z}_{1} \\
\dot{z}_{2}
\end{array}\right]+} \\
& {\left[\begin{array}{c}
\dot{q}_{2} \tan \theta \\
0
\end{array}\right]+\alpha_{1}\left[\begin{array}{cc}
1+\tan ^{2} \theta & 0 \\
0 & 1
\end{array}\right] \dot{e}_{1}+} \\
& \alpha_{2}\left[\begin{array}{c}
1+\tan ^{2} \theta \\
0 \\
0
\end{array}\right] e_{2}+\left(k_{s}+1\right) e_{2}-\left(k_{s}+1\right) e_{2}(0)+ \\
& \int_{0}^{t}\left(\left(k_{s}+1\right) \alpha_{2} e_{2}(\varsigma)+\beta \operatorname{sgn}\left(e_{2}(\varsigma)\right)\right) \mathrm{d} \varsigma
\end{aligned}
$$

where $e_{1}=\left[e_{11}, e_{12}\right]^{\mathrm{T}}=\left[\sin t-z_{1}, \frac{\pi}{4}-z_{2}\right]^{\mathrm{T}}, e_{2}=$ $\left[e_{21}, e_{22}\right]^{\mathrm{T}}=\left[\sin t+\cos t-z_{1}-\dot{z}_{1}, \frac{\pi}{4}-z_{2}-\dot{z}_{2}\right]^{\mathrm{T}}$.

In the simulation study, we chose $\alpha_{1}=1, \alpha_{2}=2, k_{s}=1$, $\beta=2$ and $z_{1}(0)=\dot{z}_{1}(0)=z_{2}(0)=\dot{z}_{2}(0)=1$. Fig. 2 shows the position tracking errors of $z(t)-z_{d}(t)$ converge to zero, and Fig. 3 shows the velocity tracking errors of $\dot{z}(t)-\dot{z}_{d}(t)$ converge to zero. At the end of the simulation, we should explain why the given signal $z_{2 d}$ is a constant. In fact, according to transformation (23), we know $z_{2}=\theta$, so the control torques ensure asymptotical tracking all the time in the unchanged yaw angle with the different velocity of flow. Hence, the practical simulation example confirms the validity of the proposed algorithm.

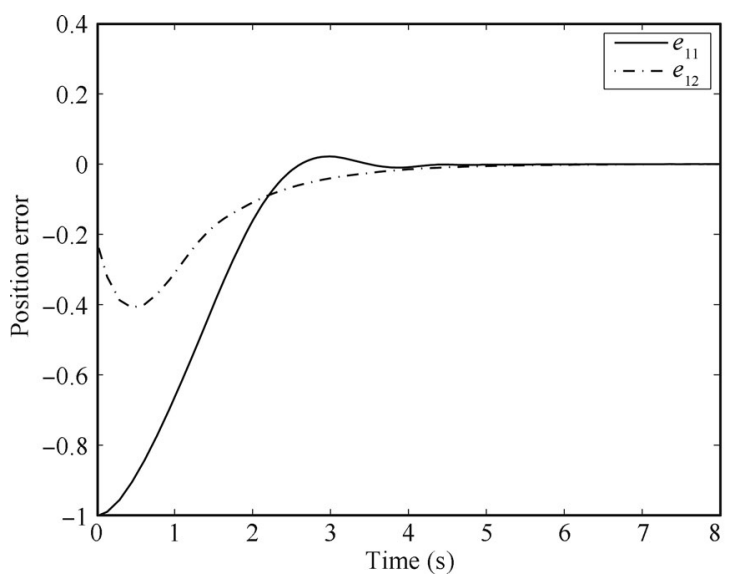

Fig. 2 The trajectories of $e_{1}$

\section{Conclusions}

In this paper, a systematic approach has been developed to design a tracking controller for the nonholonomic mechanical systems with affine constraints. The controller guarantees that the internal state tracks the desired trajectory.

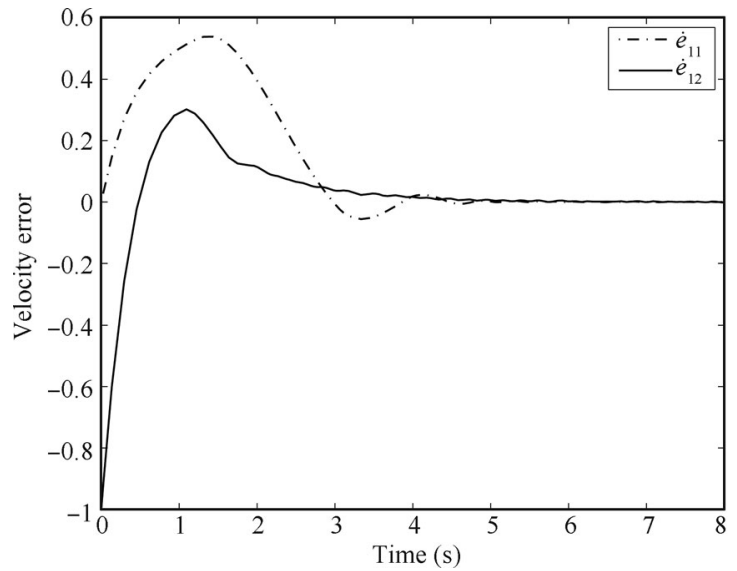

Fig. 3 The trajectories of $\dot{e}_{1}$

\section{References}

[1] Y. Q. Wu, B. Wang, G. D. Zong. Finite-time tracking controller design for nonholonomic systems with extended chained form. IEEE Transactions on Circuits and Systems II: Express Briefs, vol. 52, no. 11, pp. 798-802, 2005.

[2] X. Y. Zheng, Y. Q. Wu. Adaptive output feedback stabilization for nonholonomic systems with strong nonlinear drifts. Nonlinear Analysis: Theory, Methods and Applications, vol. 70, no. 2, pp. 904-920, 2009.

[3] Y. C. Chang, B. S. Chen. Robust tracking designs for both holonomic and nonholonomic constrained mechanical systems: Adaptive fuzzy approach. IEEE Transactions on Fuzzy Systems, vol. 8, no. 1, pp. 46-66, 2000.

[4] W. J. Dong, W. L. Xu, W. Huo. Trajectory tracking control of dynamic non-holonomic systems with unknown dynamics. International Journal of Robust and Nonlinear Control, vol. 9, no. 13, pp. 905-922, 1999.

[5] Y. Zhao, J. B. Yu, Y. Q. Wu. State-feedback stabilization for a class of more general high order stochastic nonholonomic systems. International Journal of Adaptive Control and Signal Processing, vol. 25, no. 8, pp.687-706, 2011.

[6] T. Fukao, H. Nakagawa, N. Adachi. Adaptive tracking control of a nonholonomic mobile robot. IEEE Transactions on Robotics and Automation, vol. 16, no. 5, pp. 609-615, 2000.

[7] G. L. Ju, Y. Q. Wu, W. H. Sun. Adaptive output feedback asymptotic stabilization of nonholonomic systems with uncertainties. Nonlinear Analysis: Theory, Methods and Applications, vol. 71, no. 11, pp. 5106-5117, 2009.

[8] Z. P. Wang, S. S. Ge, T. H. Lee. Robust motion/force control of uncertain holonomic/nonholonomic mechanical systems. IEEE Transactions on Mechatronics, vol. 9, no. 1, pp. 118-123, 2004.

[9] Z. J. Li, Y. N. Zhang. Robust adaptive motion/force control for wheeled inverted pendulums. Automatica, vol. 46, no. 8, pp. 1346-1353, 2010.

[10] W. J. Dong. On trajectory and force tracking control of constrained mobile manipulators with parameter dynamic. Automatica, vol. 38, no. 9, pp. 1475-1484, 2002.

[11] Z. J. Li, S. S. Ge, M. Adams. Adaptive robust outputfeedback motion/force control of electrically driven nonholonomic mobile manipulators. IEEE Transactions on Control Systems Technology, vol.16, no.6, pp.1308-1315, 2008.

[12] W. J. Dong, W. L. Xu. Adaptive tracking control of uncertain nonholonomic dynamic system. IEEE Transactions on Automatic Control, vol.46, no. 3, pp.450-454, 2001. 
[13] W. J. Dong, K. D. Kuhnert. Robust adaptive control of nonholonomic mobile robot with parameter and nonparameter uncertainties. IEEE Transactions on Robotics, vol. 21, no. 2, pp. 261-266, 2005.

[14] M. Oya, C. Y. Su, R. Katoh. Robust adaptive motion/force tracking control of uncertain nonholonomic mechanical systems. IEEE Transactions on Automatic Control, vol. 19, no. 1, pp. 175-181, 2003

[15] T. Kai. Modeling and passivity analysis of nonholonomic Hamiltonian systems with rheonomous affine constraints. Communications in Nonlinear Science and Numerical Simulation, vol. 17, no. 8, pp. 3446-3460, 2012.

[16] T. Kai, H. Kimura, S. Hara. Nonlinear control analysis on nonholonomic dynamic systems with affine constraints. In Proceedings of the 44th IEEE Conference on Decision and Control and 2005 European Control Conference, IEEE, Seville, Spain, pp. 1459-1464, 2005.

[17] T. Kai. Mathematical modelling and theoretical analysis of nonholonomic kinematic systems with a class of rheonomous affine constraints. Applied Mathematical Modelling, vol. 36, no. 7, pp. 3189-3200, 2012.

[18] B. Xian, D. M. Dawson, M. S. de Queiroz, J. Chen. A continuous asymptotic tracking control strategy for uncertain multi-input nonlinear systems. IEEE Transactions on $\mathrm{Au}$ tomatic Control, vol. 49, no. 7, pp. 1206-1211, 2004.

[19] C. Makkar, G. Hu, W. G. Sawyer, W. E. Dixon. Lyapunovbased tracking control in the presence of uncertain nonlinear parameterizable friction. IEEE Transactions on Automatic Control, vol. 52, no. 10, pp. 1988-1994, 2007.

[20] P. M. Patre, W. MacKunis, K. Dupree, W. E. Dixon. Modular adaptive control of uncertain euler-lagrange systems with additive disturbances. IEEE Transactions on Automatic Control, vol. 56, no. 1, pp. 155-160, 2011.

[21] S. S. Ge, T. H. Lee, C. J. Harris. Adaptive Neural Network Control of Robust Manipulators, Singapore: Word Scientific, 1998.
[22] H. K. Khalil. Nonlinear Systems, 3rd ed., New Jersey: Prentice Hall, 2002.

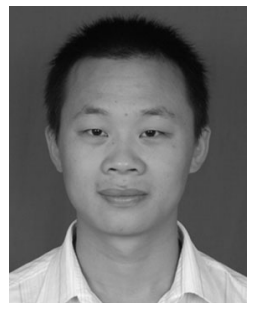

Wei Sun received his M.Sc. degree in operation research and control theory from Qufu Normal University, China in 2011. He is now a Ph. D. candidate in the School of Automation, Southeast University, China.

His research interests include nonlinear system control, adaptive theory and nonholonomic systems control.

E-mail: tellsunwei@sina.com (Corresponding author)

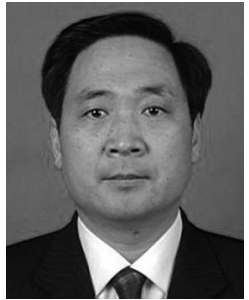

Yu-Qiang Wu received his M. Sc. degree from Qufu Normal University, China in 1988 and his Ph. D. degree from Southeast University, China in 1994. He is currently a professor in the Institute of Automation, Qufu Normal University.

His research interests include variable structure control, switching control, nonlinear system control, and process control.

E-mail: wyq@qfnu.edu.cn

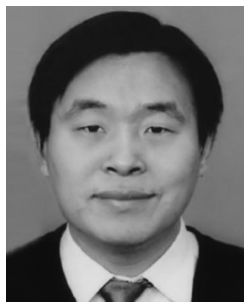

Zong-Yao Sun received his M.Sc. degree from Qufu Normal University, China in 2005, his Ph. D. degree from Shandong University, China in 2009. He is an associate professor in the Institute of Automation, Qufu Normal University.

His research interests include nonlinear control, time-delay systems and adaptive theory.

E-mail: sunzongyao@sohu.com 